\title{
State-Space Modeling and Stability Analysis for Microgrids with Distributed Secondary Control
}

\author{
Mahmuda Begum ${ }^{1}, \mathrm{Li} \mathrm{Li}^{1}$, Jianguo Zhu ${ }^{1}$ and ${\mathrm{Zhen} \mathrm{Li}^{2}}^{2}$ \\ ${ }^{1}$ Faculty of Engineering and Information Technology, University of Technology Sydney, Australia \\ ${ }^{2}$ School of Automation, Beijing Institute of Technology, China \\ Email: Mahmuda.Begum@student.uts.edu.au
}

\begin{abstract}
High penetration of renewable energies in power systems leads to the necessity of comprehensive modelling of a microgrid (MG) for its appropriate control. The distributed secondary control in the MG can be used for complementing the role of primary droop-based control. This paper presents a systematic way of developing a linearized small signal state space model with distributed secondary control as well as stability analysis of an islanded AC MG. The MG considered here, consists of three distributed generations (DGs) represented in the synchronous (DQ) reference frame. To show the effect of controller parameters on system stability, the eigenvalue analysis is presented here. The MATLAB/Simulink model of islanded MG with both primary and secondary control strategies is also developed to verify the outcomes of small-signal analysis. The simulation results show that the voltage controller simultaneously achieves the critical voltage restoration and accurate reactive power sharing.
\end{abstract}

Keywords- Distributed Control, State Space Model, Consensus Control, Autonomous Microgrid.

\section{INTRODUCTION}

Microgrid is an emerging idea for future power distribution system that empowers renewable energy integration. It generally has multiple DGs that are usually interfaced to the main grid through power electronics converters [1]. For the islanding operation of AC microgrids, two important tasks are to share the load demand among multiple parallel-connected inverters proportionately, and maintain the voltage and frequency stabilities. In future, power systems will mainly be collection of a number of interconnected microgrids where each microgrid is responsible of maintaining its own demand of energy with the neighboring microgrids in case of additional generation. Hence, the future microgrid technology will be converted to distributed ones where the generation and consumption should be arranged as a whole unit of multiple distributed microgrids, or just distributed agents. Appropriate coordination of internal components by proper control techniques is essential for a microgrid to gain such functionality.

To design the control system of autonomous microgrid effectively, lots of research works have been done over the last few decades. Thus, hierarchical control for islanded $\mathrm{MG}$ systems has been proposed for standardizing the operation and functionality of microgrid systems [2]. This hierarchical control divides the whole control structure into three layers, namely, primary, secondary and tertiary control. The decentralized droop control method has been identified as a viable solution for the primary control since it obviates the need for communications [3]. However, it exhibits the limitation of steady-state frequency and voltage deviations. Moreover, disadvantages of the primary control include its load-dependent frequency/voltage deviations, poor performance of reactive sharing and insufficient support of nonlinear loads. These problems lead to the next level of hierarchy control named secondary control. Centralized secondary controller located in the microgrid central controller is practiced to restore the frequency and voltage to their nominal values. Distributed control structures have been introduced to overcome the weakness of centralized and decentralized control methods. Distributed secondary control structures have been presented for the control of MGs to achieve system's less complexity, frequency synchronization and voltage regulation, secure active and reactive power sharing, and compensation of harmonics and unbalances. These distributed control approaches are mostly based on multi-agent system or consensus protocols [46]. Consensus-based distributed approaches have gained popularity recently, as they guarantee a good performance with a sparse communication network.

However, the dynamic performance of microgrid with the distributed secondary control may be undesirable in terms of the system damping, response time, stability margin and so on. Further, the distributed secondary control may introduce new less-damped modes to the system leading to oscillatory responses, that is, the distributed control may affect the system stability. Therefore, they need to be well tuned. To develop a control strategy for MGs and investigate its small-signal stability, first, an adequate state space model is required. There are few papers that focus on inverter-based microgrid modeling. In [7], a modeling approach which only includes VSIs and the effect of primary control is shown. Previous dynamic analysis of standalone MGs is carried out with some simplifying assumptions in the modeling of the system $[8,9]$; therefore, the analysis of voltage control is not accurate on these models. Although there are some technical papers which perform smallsignal stability analyses for the distributed secondary control [10], yet to the best of our knowledge, a mechanism analysis of the undesirable dynamic performance and the possible oscillations caused by the distributed secondary controller has not been reported before. Moreover, for practical low voltage (LV) microgrids, the impact of the line resistance (R) is not negligible and must be considered in the system.

Most of the existing approaches to the stability study of such a system are based on the transfer function [8], state-space modeling and small-signal analysis based on the linearization around an operating point and calculating the eigenvalues [10, 11], and the impedance-based method [12]. The transfer function based stability analysis does not include the impact of 
the grid impedances. On the other hand, the impedance-based and the eigenvalue-based analysis need less computation and include the impact of controller dynamics and the grid impedance; therefore, these two methods are more suitable for the stability analysis of power electronics based power systems.

The state-space modeling and the eigenvalue-based approach is a global stability analysis method that determines the system stability regardless of the source location of instability. Although many methods have been investigated to improve stability of the primary control [11], to the best of our knowledge, very few works are carried out considering the distributed secondary control in the state space modelling for detailed small-signal stability analysis. Motivated by the aforementioned limitations, this paper focuses on integrated modeling, analyzing and stabilizing of microgrids with the distributed secondary control. The main contributions of this paper are:

$>\quad$ A unified small-signal dynamic model of the proposed distributed secondary voltage controller, taking into account also the line resistance in the model.

$>\quad$ The proposed voltage controller can keep accurate reactive power sharing after voltage restoration.

A detailed impact assessment of the distributed controllers on the microgrid dominant oscillatory modes based on the small-signal dynamic model.

$>$ A precise proof for the realization of the desired control objectives of the proposed distributed controllers based on time-domain simulation.

The rest of the paper is organized as follows: In Section II, the proposed distributed voltage controller is presented. In Section III, the state space model of a test microgrid considering distributed secondary controller is explained. Modeling of the network and load is also given in this section. In Section IV, the simulation for a MG with three DGs is shown, and lastly, the conclusion is given in Section V.

\section{DISTRIBUTED SECONDARY CONTROL}

Fig. 1 shows the diagram of a basic structure of distributed control system where primary and secondary controls are implemented in each DG unit. In this section, some basic terminologies of consensus control [4] are given, which will be used in the design of proposed distributed secondary control. Based on these, the secondary control objectives are proposed and then a distributed secondary control law is designed for the MG to restore the deviated voltage to their reference value while maintaining the accurate reactive power sharing.

\section{A. Consensus Control Basics}

Consensus problem is one of the most basic and challenging problems in cooperative control. It is assumed that there are

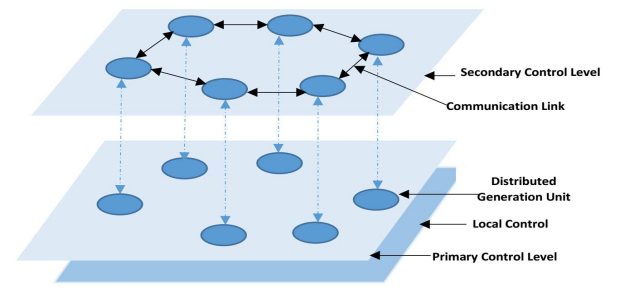

Fig. 1. Basic structure of distributed control techniques multiple agents on a network. This network is generally modeled by a graph connection of nodes (representing the agents) and edges (representing the interactions between agents). If all the agents on a network converge to a common state, the multi-agent system resolves a consensus problem or has a consensus property, and the common state is entitled group decision value or consensus state.

\section{B. Graph Theory}

A directed graph (digraph) $G=\left(N_{G}, E_{G}\right)$ with a set of $N$ nodes, $N_{G}=\{1,2,3,4 \ldots \ldots . N\}$, a set of edges $E_{G} \subset N_{G} \times N_{G}$ and an adjacency matrix $A_{G}=\left(a_{i j} \geq 0\right) \in R^{N \times N}$ (where $a_{i j}=$ 1 if the $i^{\text {th }}$ node is connected to the $j^{\text {th }}$ node and otherwise $\left.a_{i j}=0\right)$ is introduced here. Each node denotes an agent, and each edge $(i, j)$ (pointing from $j$ to $i$ ) denotes that data can flow from $j$ to $i$ associated with $a_{i j}$. Define the neighbors of node $i$ as $N_{i}=\left\{j \in N:(i, j) \in E_{G}\right\}$. Thus, under this description, an agent/node $i$ only has access to the information from its neighbors in $N_{i}$.

Let each agent (node) be a single-state system described by $\dot{x}_{l}=u_{i}$ where $u_{i}$ is the input as a function of the $i^{t h}$ agent's neighboring state $x_{j}, j \in N_{i}$. The usual practice is to take on the following consensus protocol:

$$
\dot{x}_{\imath}=u_{i}=\sum_{j \in N_{i}} a_{i j}\left(x_{j}-x_{i}\right)
$$

\section{Consensus-based Distributed Secondary Control}

In this paper, a basic microgrid comprising of three DG units is considered and it can also be applicable for more DG units in parallel connection. Each DG unit is linked to the respective load and interconnected with neighboring DG units through feeder lines. Coordination of the primary controllers can be achieved by considering the droop control as in Fig. 2 . The following equation represents the droop controller of the $i^{t h}$ DG for voltage regulation:

$$
v_{i}=v_{r e f}-n_{q} Q_{i}
$$

where $Q_{i}$ is the reactive power of the $i^{\text {th }}$ DG units, $n_{q}$ is the voltage droop gain, and $v_{r e f}$ is the nominal voltage. Thus, the secondary control is achieved by choosing the proper control input $u_{i}$ to adjust the individual voltage magnitude $v_{i}$ to the reference $v_{\text {ref }}$ synchronously with all the agents acting as a group. Therefore, the consensus based distributed secondary control signal for the $i^{\text {th }}$ DG can be written as

$$
h_{i}=-C_{v i} \int\left[\sum_{j \in N_{i}}\left(v_{\text {diff } i}-v_{\text {diff } j}\right)+g_{i}\left(v_{\text {diffi } i}-v_{\text {diff }}\right)\right] \ldots
$$

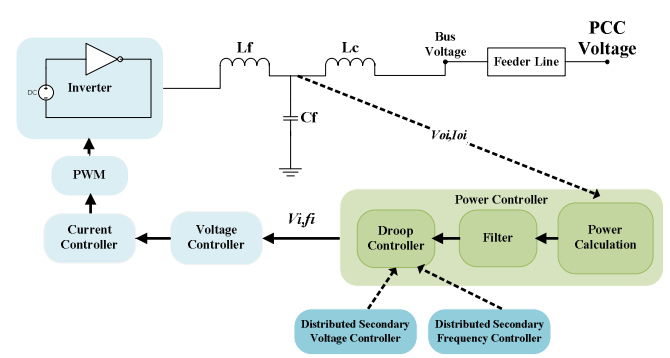

Fig. 2. Block diagram for a DG unit with droop controller 


$$
\tau_{i}=-C_{Q i} \int\left[\sum_{j \in N_{i}} a_{i j}\left(\frac{Q_{i}}{Q_{\text {imax }}}-\frac{Q_{j}}{Q_{j \max }}\right)\right] \ldots
$$

where $h_{i}$ is the secondary control signal for voltage restoration and $\tau_{i}$ is the secondary control signal for reactive power sharing. Here, $v_{\text {diff }}$ calculated at the point of common coupling (PCC) as in Fig. 1 and $v_{\text {diffi }}$ are given through the following equations

$$
\begin{aligned}
& v_{\text {diff }}=k_{p}\left(v_{\text {ref }}-v_{P C C}\right)+k_{I} \int\left(v_{\text {ref }}-v_{P C C}\right) d t . \\
& v_{\text {diffi }}=k_{p}\left(v_{\text {ref }}-v_{\text {odi }}\right)+k_{I} \int\left(v_{\text {ref }}-v_{\text {odi }}\right) d t .
\end{aligned}
$$

Note that, in (2), $g_{i}=1$ if the $i^{\text {th }}$ DG has direct communication with the controller at PCC and otherwise $g_{i}=$ 0 . Combining the secondary control signal in (2) and (3) with the primary control signal in (1), the resulting inverter voltage reference can be shown as below,

$$
v_{\text {refi }}=v_{i}+h_{i}+\tau_{i}
$$

\section{Small-Signal State Space Model of Autonomous AC MICROGRID}

The modeling approach presented in this paper divides the whole system into three major sub-modules: inverter, network and load [7].

\section{A. State-Space Model of Individual Inverter}

Each inverter is modeled on its individual reference frame whose rotation frequency is set by its local power sharing controller. The inverter model includes the power sharing controller dynamics, output filter dynamics, coupling inductor dynamics and voltage and current controller dynamics (Fig. 2). Small-signal modeling of parallel-connected inverters is carried out considering the network dynamics. Here, state equations of the network and loads are represented on the reference frame of one of the individual inverters. This reference frame is considered as the common reference frame. All the other inverters are translated to this common reference frame using the transformation technique [11] depicted in Fig. 3 and defined in (6). Here, the axis set is the common reference frame rotating at a frequency $w_{c o m}$, whereas axes $(d-q)_{i}$ and $(d-q)_{j}$ are the reference frame of $i^{\text {th }}$ and $j^{\text {th }}$ inverters rotating at $\omega_{i}$ and $\omega_{j}$, respectively.

$$
\text { Now, }\left[f_{D Q}\right]=\left[T_{i}\right]\left[f_{d q}\right]
$$

$\left[T_{i}\right]=\left[\begin{array}{cc}\cos \left(\delta_{i}\right) & -\sin \left(\delta_{i}\right) \\ \sin \left(\delta_{i}\right) & \cos \left(\delta_{i}\right)\end{array}\right]$

where $\delta_{i}$ is the angle of the reference frame of $i^{\text {th }}$ inverter with respect to the common reference frame. A complete state-space

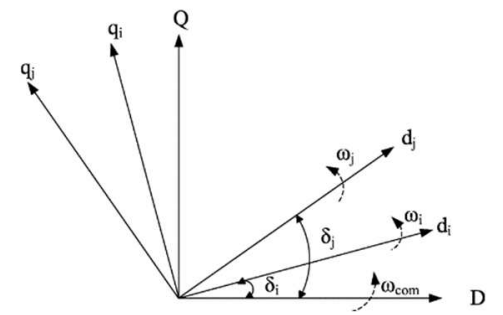

Fig. 3. Reference Frame Transformation [7] small-signal model of the inverter can be obtained by combining the state-space models of the power controller, voltage controller, current controller and output LCL filter. The details of internal modelling of all these modules can be found in [7] considering the primary control. It is to be noted that in the equations of the following sections, the three-phase voltages and currents are represented as vectors in $D-Q$ frame whereas the other variables such as real and reactive powers and angles are scalars.

\section{B. State-Space Model of Individual Inverter with Distributed Secondary Voltage Control (DSVC)}

According to [7], the complete small-signal linearized statespace model of an individual inverter unit in the common reference frame considering the primary control (droop control) only can be written as below:

$\left[\Delta \dot{x}_{\text {invi }}\right]=\left[A_{\text {invi }}\right]\left[\Delta x_{\text {invi }}\right]+\left[B_{\text {invi }}\right]\left[\Delta v_{b D Q i}\right]+\left[B_{\text {iwcom }}\right]\left[\Delta \omega_{\text {com }}\right]$

$\left[\begin{array}{c}\Delta \omega_{i} \\ \Delta i_{0 D Q i}\end{array}\right]=\left[\begin{array}{c}C_{I N V \omega i} \\ C_{I N V c i}\end{array}\right]\left[\Delta x_{i n v i}\right]$

where $\Delta x_{i n v i}=\left[\Delta \delta_{i}, \Delta P_{i}, \Delta Q_{i}, \Delta \emptyset_{d q i}, \Delta \gamma_{d q i}, \Delta i_{l d q i}, \Delta v_{0 d q}, \Delta i_{0 d q}\right]^{T}$

Similarly, small signal linearized state-space model of the individual inverter unit in the common reference frame considering the proposed distributed secondary control (from (1)-(6)) can be written as below:

$\left[\Delta \dot{x}_{\text {invi }}\right]=\left[A_{\text {invi }}\right]\left[\Delta x_{\text {invi }}\right]+\left[B_{\text {invi }}\right]\left[\Delta v_{b D Q i}\right]+$

$\left[B_{i w c o m}\right]\left[\Delta w_{\text {com }}\right]+\sum_{j \in N_{i}}\left[F_{\text {invij }}\right]\left[\Delta x_{i n v j}\right]+$

$\left[H_{\text {invi }}\right]\left[\Delta v_{\text {PCCref }}\right]$.

$\left[\begin{array}{c}\Delta \omega_{i} \\ \Delta i_{0 D Q i}\end{array}\right]=\left[\begin{array}{c}C_{I N V \omega i} \\ C_{I N V c i}\end{array}\right]\left[\Delta x_{i n v i}\right]$

In (9), parameter matrices $F_{i n v i j}$ represents the correlations between $D G_{i}$ and the neighbor $D G_{j}$ provided by the distributed secondary control. In (9) \& (10), the state variables of each $D G$ unit are considered as $\Delta x_{i n v i}=$

$\left[\begin{array}{lllllllllll}\Delta \delta_{i} & \Delta P_{i} & \Delta Q_{i} & \Delta h_{i} & \Delta \tau_{i} & \Delta \emptyset_{d q i} & \Delta \gamma_{d q i} & \Delta i_{l d q i} & \Delta v_{0 d q} & \Delta i_{0 d q} \Delta \sigma_{i}\end{array}\right]^{T}$

Here, the equation for new states of secondary controller can be written as

$$
\begin{aligned}
& \Delta h_{i}=C_{v i} \int\left[\sum_{j \in N_{i}} a_{i j}\left(\Delta v_{\text {diff } j}-\Delta v_{\text {diffi }}\right)+g_{i}\left(\Delta v_{\text {diff }}-\Delta v_{\text {diffi }}\right)\right] \ldots \ldots \\
& \Delta \tau_{i}=C_{Q i} \int\left[\sum_{j \in N_{i}} a_{i j}\left(\frac{Q_{j}}{Q_{j \max }}-\frac{Q_{j}}{Q_{j \max }}\right)\right] \ldots \ldots \ldots \ldots \ldots \ldots \ldots \ldots \ldots \ldots \ldots \ldots \ldots \ldots \ldots \ldots \ldots \ldots
\end{aligned}
$$

Letting $\epsilon=\int\left(v_{r e f}-v_{P C C}\right) d t, \dot{\epsilon}=v_{r e f}-v_{P C C}$, we have

$\dot{\Delta} \epsilon=-\Delta v_{P C C}$.

Similarly, let $\sigma_{i}=\int\left(v_{r e f}-v_{o d i}\right) d t, \dot{\sigma}_{l}=v_{r e f}-v_{o d i}$ to get

$$
\dot{\Delta \sigma_{l}}=-\Delta v_{\text {odi }}
$$

\section{Combined State-Space Model of all the Inverters}

In this paper, three DGs are considered in the proposed model. Now, by combining (9)-(14) for 3 inverters, the combined small-signal model of all the inverter units is obtained, as shown below:

$$
\begin{array}{r}
{\left[\Delta \dot{x}_{i n v}\right]=\left[A_{i n v}\right]\left[\Delta x_{i n v}\right]+\left[B_{i n v}\right]\left[\Delta v_{b D Q}\right]+} \\
{\left[B_{\text {wcom }}\right]\left[\Delta w_{\text {com }}\right]+\left[H_{\text {inv }}\right]\left[\Delta v_{P C C r e f}\right] \ldots \ldots \ldots \ldots \ldots \ldots \ldots \ldots \ldots \ldots . . .}
\end{array}
$$




$$
\left[\begin{array}{c}
\Delta \omega \\
\Delta i_{0 D Q}
\end{array}\right]=\left[\begin{array}{c}
C_{I N V \omega} \\
C_{I N V c}
\end{array}\right]\left[\Delta x_{i n v}\right]
$$

The modelling approach in this work is to form a sub-model of all the individual DG inverters and combine them with the network and individual load models.

\section{Network Sub-module Model}

Small-signal linearized state space model of the network sub-module comprising of two RL type lines can be given in the common reference frame [7] as shown below,

$$
\left[\Delta \iota_{\text {llneDQ }}^{\cdot}\right]=\left[A_{\text {net }}\right]\left[\Delta i_{\text {lineDQ }}\right]+\left[B_{1 \text { net }}\right]\left[\Delta v_{b D Q}\right]+
$$

$\left[B_{2 n e t}\right][\Delta w]$

Here, $\Delta w=\Delta w_{\text {com }}$;

$$
\begin{gathered}
A_{\text {net }}=\left[\begin{array}{cc}
A_{\text {net } 1} & 0 \\
0 & A_{\text {net } 2}
\end{array}\right] ; B_{1 \text { net }}=\left[\begin{array}{ccc}
B_{1 \text { net } 1} & -B_{1 \text { net } 1} & 0 \\
0 & B_{1 \text { net } 2} & -B_{1 \text { net } 2}
\end{array}\right] \\
B_{1 \text { net } i}=\left[\begin{array}{cc}
\frac{1}{L_{\text {linei }}} & 0 \\
0 & \frac{1}{L_{\text {linei }}}
\end{array}\right] ; B_{2 \text { net }}=\left[\begin{array}{l}
B_{2 \text { net } 1} \\
B_{2 \text { net } 2}
\end{array}\right] ; B_{2 \text { net } i}=\left[\begin{array}{c}
I_{\text {lineQi }} \\
-I_{\text {lineDi }}
\end{array}\right] ;
\end{gathered}
$$

\section{E. Load Sub-module Model}

Small-signal linearized state-space model of the RL type load sub-module can be written [7] as in (18)

$$
\begin{aligned}
& {\left[\Delta \iota_{\text {loadDQ }}\right]=\left[A_{\text {load }}\right]\left[\Delta i_{\text {loadDQ }}\right]+\left[B_{1 \text { load }}\right]\left[\Delta v_{\text {bDQ }}\right]} \\
& +\left[B_{2 \text { load }}\right][\Delta w] \\
& \text { where, } A_{\text {load }}=\left[\begin{array}{cc}
A_{\text {load } 1} & 0 \\
0 & A_{\text {load } 2}
\end{array}\right] \text {; } \\
& B_{1 \text { load }}=\left[\begin{array}{cc}
B_{1 \text { load } 1} & 0 \\
0 & B_{1 \text { load } 2}
\end{array}\right] \\
& B_{1 \text { loadi }}=\left[\begin{array}{cc}
\frac{1}{L_{\text {loadi }}} & 0 \\
0 & \frac{1}{L_{\text {loadi }}}
\end{array}\right] ; \quad B_{\text {2loadi }}=\left[\begin{array}{c}
I_{\text {loadQi }} \\
-I_{\text {load } i}
\end{array}\right]
\end{aligned}
$$

The line resistance $(\mathrm{R})$ cannot be neglected in the model of LV microgrids. Denote the deviation of $i_{o i}$, of all the DG units as $\Delta i_{o D Q}$. Then the bus voltage, $\Delta v_{b D Q}$ is represented by

$$
\left[\Delta v_{b D Q}\right]=R_{N}\left(M_{i n v}\left[\Delta i_{o D Q}\right]+M_{N E T}\left[\Delta i_{\text {lineDQ }}\right]+\right.
$$$$
\left.M_{\text {Load }}\left[\Delta i_{\text {loadDQ }}\right]\right)
$$

Similarly, Vpcc is the deviation of the PCC voltage, and can be expressed in terms of $i_{0 d q}, i_{\text {linedq }}$ and $i_{\text {loaddq }}$ like as in (19). The complete microgrid small-signal state-space model and the system matrix can be obtained by using the individual subsystem models given by (15)-(19) as in (20). The system dynamics and stability can be then evaluated based on the eigen-structure of system matrix as given in the following parts.

$$
\left[\begin{array}{c}
\Delta \dot{X}_{\text {lnv }} \\
\Delta u_{\text {lne }} \\
\Delta u_{\text {load }} \\
\Delta \in
\end{array}\right]=A_{M G}\left[\begin{array}{c}
\Delta X_{\text {inv }} \\
\Delta i_{\text {line }} \\
\Delta i_{\text {load }} \\
\Delta \in
\end{array}\right] .
$$

\section{Simulation AND Result}

\section{A. Test System}

A test microgrid model of three inverter based DG units including the local loads, is presented in Fig. 4. The microgrid considered here is a $311 \mathrm{~V}$ and $50 \mathrm{~Hz}$ system and is simulated in MATLAB. The steady-state operating point conditions are obtained from a MATLAB/Simulink time-step simulation of the system. DG unit 1 and DG unit 3 are connected to load1 and load3 respectively through the coupling inductance $L_{c}(0.1 \mathrm{mh})$ as in Fig. 1. The microgrid is functioned in the autonomous mode, and each three-phase load is represented by a series RL branch and each feeder line is modeled by a lumped, series RL branch in each phase. Tables I, II and III provide the system and the DG primary and secondary control parameters, respectively.

It is assumed that DG units communicate with neighbors through the directed graph as in Fig. 4. For the voltage restoration problems, the voltage reference is considered to be the DG2 unit outputs. Fig. 4 also indicates that (i) all DGs like $D G 1 \sim D G 3$ are root nodes, and (ii) $D G 2$ is the only root node with the reference value and the pinning gain of $g_{2}=1$. The results are divided into two parts. One is eigenvalue analysis and the other is time domain simulation. The steps of the simulations can be depicted in Fig. 5.

\section{B. Eigenvalue Analysis}

This subsection presents the system dynamics and stability analysis results for the test microgrid under the proposed DSVC. The MATLAB function eig $(\cdot)$ is employed to calculate the eigenvalues of the system matrix $A_{M G}$. According to the established small-signal model in the previous section, the resulting eigenvalues considering DSVC are shown in Fig. 6 for stable operation. As the eigenvalues of high and intermediate frequency modes have weak influence on the system stability [7], in this paper, the focus of analysis is on the low-frequency modes. The dominant oscillatory modes are shown in Fig. 6. Fig. 7 shows the eigenvalues (for both without and with DSVC), which indicates that the secondary control (i) considerably changes the shaping of the eigenvalues on the complex plane and (ii) creates a new pair of less-damped modes yielding more oscillatory system responses as compared with the primary

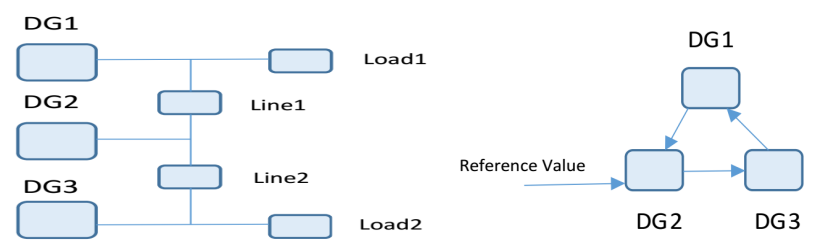

Fig. 4. Simulation diagram of the microgrid test model and the communication diagraph.

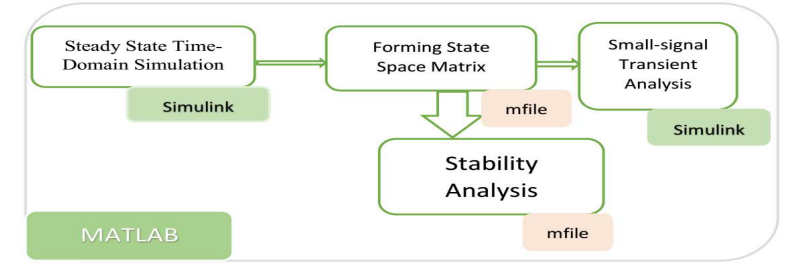

Fig. 5. Steps of stability analysis process in MATLAB

responses. Therefore, the system stability is highly affected by the secondary control.

1) Participation Factors: Participation factor is the multiplication of corresponding element in the right and left eigenvectors of the state matrix to measure the association between the state variables and the modes. Participation factors 
suggest that in case of the primary droop control, the lowfrequency modes are mainly affected by the states of active power droop controller (1), i.e., $\Delta \delta_{i}$ and $\Delta P_{i}$ [7]. The participation factor relating the $k^{\text {th }}$ state variable to the $i^{\text {th }}$ eigenvalue, in a system with $n$ eigenvalues, is defined as

$$
p_{k i}=\frac{\lambda_{i}}{a_{k k}}=\frac{\left|v_{k i}\right|\left|\omega_{k i}\right|}{\sum_{k=1}^{n}\left|v_{k i}\right|\left|\omega_{k i}\right|}
$$

TABLE I. PARAMETERS FOR THE INVERTERS USED IN THE TEST SYSTEM

\begin{tabular}{|c|c|c|c|}
\hline Description & Parameter & Value & Unit \\
\hline \multicolumn{4}{|c|}{ "Microgrid Model Parameters } \\
\hline "DC Voltage Value & 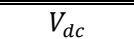 & 700 & "V \\
\hline Reference Voltage & $v_{\text {ref }}$ & 311 & V \\
\hline Reference Frequency & $f_{\text {ref }}$ & 50 & $\mathrm{~Hz}$ \\
\hline Resistance of Filter Inductor & $R_{f}$ & 0.1 & $\Omega$ \\
\hline Inductance of Filter Inductor & $L_{f}$ & 1.35 & $\mathrm{mH}$ \\
\hline Capacitance of Filter Inductor & $C_{f}$ & 50 & $\mu \mathrm{F}$ \\
\hline $\begin{array}{l}\text { Resistance of Coupling } \\
\text { Inductor }\end{array}$ & $R_{c}$ & 0.03 & $\Omega$ \\
\hline $\begin{array}{l}\text { Inductance of Coupling } \\
\text { Inductor }\end{array}$ & $L_{C}$ & 0.1 & $\mathrm{mH}$ \\
\hline \multicolumn{4}{|c|}{ "Voltage Controller Parameters } \\
\hline Proportional Gain & $K_{p v}$ & 0.05 & \\
\hline Integral Gain & $K_{i v}$ & 390 & \\
\hline Feed Forward Gain & $\mathrm{F}$ & 0.75 & \\
\hline \multicolumn{4}{|c|}{ Current Controller Parameters } \\
\hline Proportional Gain & $\bar{K}_{p c}$ & 10.2 & \\
\hline Integral Gain & $K_{i c}$ & $16 \mathrm{e} 3$ & \\
\hline
\end{tabular}

TABLE II. Line and Load Data used in the Test System

\begin{tabular}{cccccc}
\hline \hline \multicolumn{3}{c}{ Line Data } & \multicolumn{3}{c}{ Load Data } \\
\hline \hline No. & $\mathrm{R}(\Omega)$ & $\mathrm{L}(\mu \mathrm{H})$ & No. & $\mathrm{R}(\Omega)$ & $\mathrm{L}(\mathrm{mH})$ \\
Line 1 & 0.23 & 318 & Load1 & 40 & 15 \\
Line 2 & 0.30 & 312 & Load2 & 50 & 22
\end{tabular}

TABLE III. Secondary Controller Parameters

\begin{tabular}{|l|c|c|}
\hline \hline \multicolumn{2}{|c|}{ Controller Parameters } & Values \\
\hline \hline Proportional Gain & $K_{P}$ & 0.001 \\
\hline Integral Gain & $K_{I}$ & 50 \\
\hline Voltage Gain & $C_{V}$ & 100 \\
\hline Reactive Power Gain & $C_{Q}$ & 28 \\
\hline
\end{tabular}

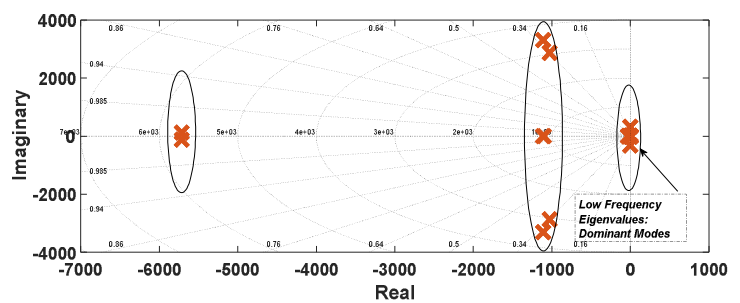

Fig. 6. Eigenvalues of system matrix considering DSVC for stable operation

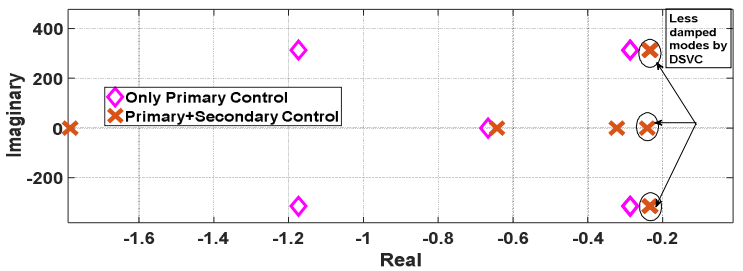

Fig. 7. Dominant modes for both primary and considering DSVC for stable operation where $\lambda_{i}$ is the $i^{\text {th }}$ eigenvalue, $a_{k k}$ is the $k^{\text {th }}$ diagonal element of the system matrix, and $\omega_{k i}$ and $v_{k i}$ are the $k^{t h}$ elements of the left and right eigenvectors associated with $\lambda_{i}$ [7]. Table IV compares the most dominant oscillatory modes of the microgrid with and without considering the secondary control. It is indicated that the secondary control introduces three new lessdamped eigenvalues, i.e., modes 7, 8 and 9.

TABLE IV. Secondary Controller Most Dominant Modes with and without Secondary Control

\begin{tabular}{|c|c|c|c|c|c|c|c|c|}
\hline \multicolumn{9}{|c|}{ Eigenvalues Comparison } \\
\hline \multirow{2}{*}{$\begin{array}{ll}\frac{0}{0} & \\
\sum^{\prime} & \infty\end{array}$} & \multicolumn{5}{|c|}{ primary control+secondary control } & \multicolumn{3}{|c|}{ primary control } \\
\hline & 1,2 & 3,4 & 5,6 & 7,8 & 9 & 1,2 & 3,4 & 5,6 \\
\hline Real & -52 & -48 & -32 & -1 & -0.5 & -59 & -49 & -39 \\
\hline Imag & \pm 89.3 & \pm 40 & \pm 79.7 & \pm 2.2 & 0 & \pm 98.2 & \pm 45 & \pm 32.6 \\
\hline
\end{tabular}

2) Eigenvalue Sensitivity to Secondary Control Parameters on System Stability: Fig. 8 shows the traces of low-frequency modes of the system as functions of $K_{I}, K_{P}, C_{V}$ and $C_{Q}$. Fig. 8(a) and Fig. 8(b) shows changes in the damping of the "least damped" mode when $\mathrm{K}_{\mathrm{I}}$ and $\mathrm{K}_{\mathrm{P}}$ both vary from 0 to 60 and $1 \mathrm{e}-$ 4 to $1 \mathrm{e}-1$ respectively. These variations mainly affect mode 9 . Fig. 8(c) shows that $C_{Q}$ has also opposite effects on the damping. $\mathrm{C}_{\mathrm{V}}$ has much less effect on the stability, and that's why it is not shown here. The above analysis provides general guidelines for the secondary control parameters design.

\section{Time-domain Simulation}

To verify the performance of the proposed secondary controller, a set of time-domain simulation studies in the Matlab/Simpower system platform are also carried out.

1) Case 1: Performance of DSVC to reach the nominal values: The performance of primary droop control is shown in

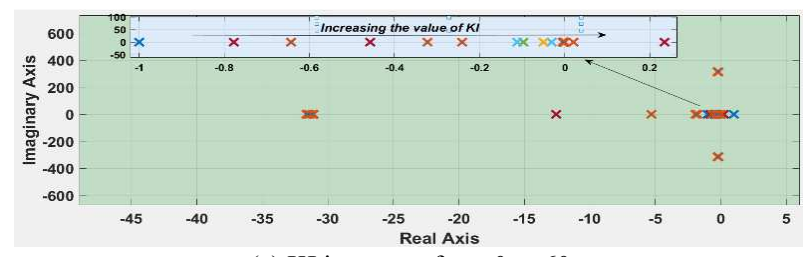

(a) $\mathrm{KI}$ increases from 0 to 60

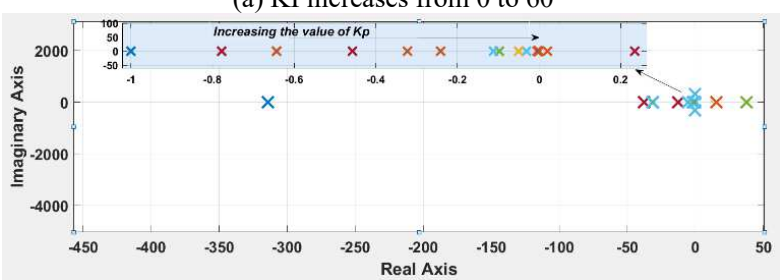

(b) KP increases from $1 \mathrm{e}-4$ to $1 \mathrm{e}-1$

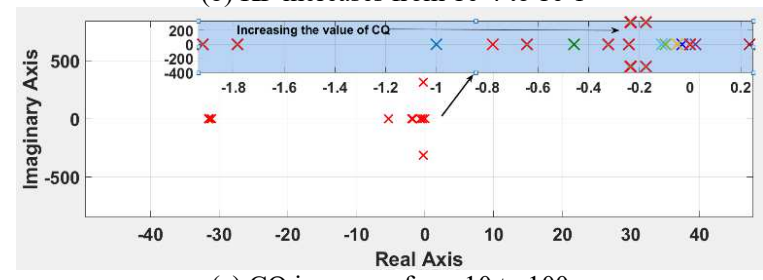

(c) CQ increases from 10 to 100

Fig. 8. Traces of low frequency modes 
Figs. 9(a) and (c). Fig. 9(a) indicates due to the droop function in the primary control, the voltage amplitudes of 3 DGs fall down to different values $(\mathrm{DG} 1=278 \mathrm{~V}, \quad \mathrm{DG} 2=269 \mathrm{~V}$, DG3 $=267 \mathrm{~V})$. It is clear that the voltages deviate from their reference values of $311 \mathrm{~V}$; hence, they need to be restored to their reference values in the secondary control layer. When our proposed distributed secondary control is applied, voltages can be quickly restored to their reference values, which is shown in Fig. 9(b). Moreover, Fig. 9(c) indicates that under the primary control the reactive power sharing is not accurate while the proposed DSVC imposes accurate reactive power sharing with the ratios of $Q_{1}$ to $Q_{3}$ being 4:2:1 as in Fig. 9(d).

Case 2: Performance of DSVC with step load change after reaching nominal values: To show the performance of DSVC with the load change, the simulation can be divided into 2 stages: Stage1 (2 - 4s): Constant load Loadc $(\mathrm{R}=20 \Omega$ and $\mathrm{L}=10 \mathrm{mH})$ is added to DG3; Stage2 (4-5s): Loadc is removed from $D G 3$. The result shows that the designed distributed secondary controller can eliminate the voltage deviation caused by the primary control. The steady state voltages of the three DGs remain at $311 \mathrm{~V}$ in Fig. 10(a) no matter the new constant load Load2 is connected to or disconnected from DG3, even though there are transient deviations. The simulation results also show that the DGs can also maintain a good reactive power sharing accuracy in Fig.10(b), no matter the additional load is

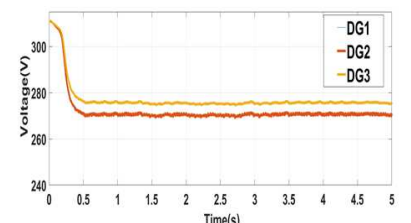

(a)

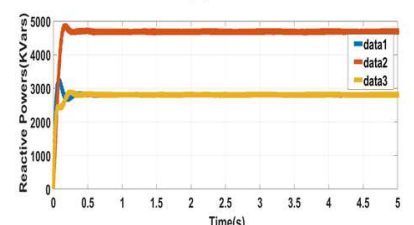

(c)

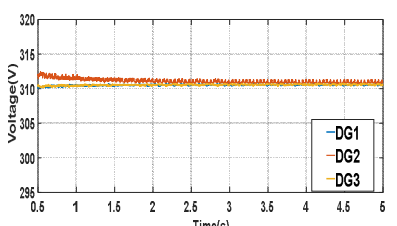

(b)

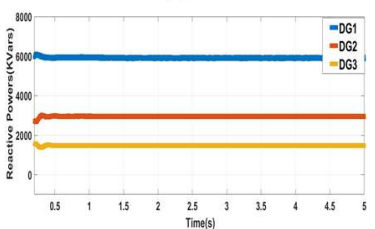

(d)
Fig. 9. Outputs of three DGs for Case 1, voltage outputs (a) without and (b) with DSVC and reactive power sharing (c) without and (d) with DSVC

connected to or disconnected from the system.

\section{CONCLUSION}

As mentioned in the introduction, there are limited approaches to solving voltage restoration problems in a distributed way. This paper presents a distributed secondary

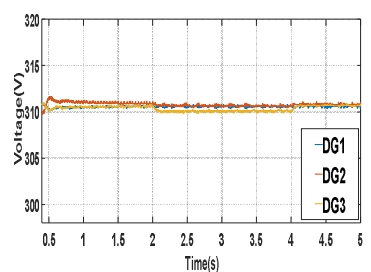

(a)

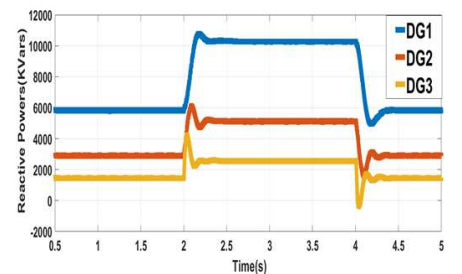

(b)
Fig. 10. Outputs of three DGs for Case 2, (a) voltage outputs (b) reactive power sharing with DSVC control method, based on the concept of the consensus control, for islanded LV microgrids to complement the primary control function and simultaneously (i) restore the system nominal voltage, (ii) provide accurate reactive power sharing among DG units. A unified small-signal dynamic model of the microgrid has been developed considering the effect of resistive line network. A small-signal stability analysis has revealed that (i) the distributed secondary controller may introduce new less damped modes to the system, (ii) the system dynamic performance is still undesirable even with carefully tuned control parameters, different load conditions and different sparse communication network topologies, and (iii) the distributed secondary controllers of all the DG units significantly participate in the newly introduced less-damped modes. The performance of the introduced concepts/methods have been evaluated and verified based on eigen-analysis and digital time-domain simulation of a microgrid test system. Based on the simulation results for the proposed small-signal dynamic model, the distributed optimal controller is considered a possibly suitable solution for effectively enhancing the system stability which is our future work.

\section{REFERENCES}

[1] M. S. Mahmoud, S. Azher Hussain, and M. A. Abido, "Modeling and control of microgrid: An overview," Journal of the Franklin Institute, vol. 351, pp. 2822-2859, 2014.

[2] A. Bidram and A. Davoudi, "Hierarchical Structure of Microgrids Control System," IEEE Transactions on Smart Grid, vol. 3, pp. 1963-1976, 2012.

[3] Z. Ahmad and S. N. Singh, "DROOP Control Strategies of Conventional Power System Versus Microgrid Based Power Systems - A Review," pp. 1499-1504, 2015.

[4] M. Begum, M. Abuhilaleh, L. Li, and J. Zhu, "Distributed secondary voltage regulation for autonomous microgrid," in 2017 20th International Conference on Electrical Machines and Systems (ICEMS), 2017, pp. 1-6.

[5] N. M. Dehkordi, N. Sadati, and M. Hamzeh, "Fully Distributed Cooperative Secondary Frequency and Voltage Control of Islanded Microgrids," IEEE Transactions on Energy Conversion, vol. 32, pp. 675685, 2017.

[6] Z. Yu, Q. Ai, J. Gong, and L. Piao, "A Novel Secondary Control for Microgrid Based on Synergetic Control of Multi-Agent System," Energies, vol. 9, p. 243, 2016 2016-04-14 2016.

[7] N. Pogaku, M. Prodanovic, and T. C. Green, "Modeling, Analysis and Testing of Autonomous Operation of an Inverter-Based Microgrid," IEEE Transactions on Power Electronics, vol. 22, pp. 613-625, 2007.

[8] M. Amin and M. Molinas, "Small-Signal Stability Assessment of Power Electronics Based Power Systems: A Discussion of Impedance- and Eigenvalue-Based Methods," IEEE Transactions on Industry Applications, vol. 53, pp. 5014-5030, 2017.

[9] F. D. Mohammadi, H. Keshtkar, and A. Feliachi, "State Space Modeling, Analysis and Distributed Secondary Frequency Control of Isolated Microgrids," IEEE Transactions on Energy Conversion, pp. 1-1, 2017.

[10]X. Wu and C. Shen, "Distributed optimal control for stability enhancement of microgrids with multiple distributed generators," IEEE Transactions on Power Systems, pp. 1-1, 2017.

[11]X. Chen, C. Zhang, Q. Huang, and M. Ofori-Oduro, "Small-Signal Modeling and Analysis of Grid-Connected Inverter with Power Differential Droop Control," Mathematical Problems in Engineering, 2016 2016-08-18 2016.

[12]J. Sun, "Impedance-Based Stability Criterion for Grid-Connected Inverters," IEEE Transactions on Power Electronics, vol. 26, pp. 30753078, 2011. 